\title{
Information Communication Technology: Assisting Social and Economic Sustainability Represented by a Fitness Landscape Model
}

\author{
Clarence S. Bayne and Raafat George Saadé \\ DISMIS Department, JMSB, Concordia University, \\ Montreal, Canada
}

bayne@alcor.concordia.ca; rsaade@imsb.concordia.ca

\begin{abstract}
The institute for Community Entrepreneurship and Development (ICED) at JMSB Concordia University, has a long history of involvement in the practice of community development and social entrepreneurship within the Cree, Black and other minority communities. The visible minority communities account for a significant proportion of the populations in Metropolitan areas across Canada. But their community organizations and businesses are small and exist outside the mainstream of Canadian growth and prosperity. These types of businesses lack access to the kind of knowledge and updates in knowledge essential to keeping pace with the growth in capacity and ingenuity necessary for making them competitive.

The gaps in ingenuity and management skills that retard the development of these micro-family based businesses can be removed by addressing two problems. The first involves modifying traditional economics and managements principles and concepts and ways of teaching them so that they are more sensitive to the scale of operations of "below the radar organizations" and businesses entering the national market via these "cracks in the market". The second relate to the use of communication technology for creating a network system of activity centers that receive, process (evaluate, use and update knowledge or ingenuity) and distribute information on a continuous and real time basis to these businesses and organizations. In order to better understand this in a social and economic context, we have drawn on the concept of the fitness landscape within the framework of a self-adaptive cultural change model. We argue that the fundamental reason for social change and adaptation is to optimize social and economic well being on a continuous and sustained basis and can be facilitated through the use of a portal designed to meet the fitness landscape theory. In this article, we discuss the theory of fitness landscape in context, demonstrate the portal, and present the results of a survey that aims at identifying critical success factors.
\end{abstract}

Keywords: fitness landscape, social sustainability, economic sustainability, community development

Material published as part of this publication, either on-line or in print, is copyrighted by the Informing Science Institute.

Permission to make digital or paper copy of part or all of these works for personal or classroom use is granted without fee provided that the copies are not made or distributed for profit or commercial advantage AND that copies 1) bear this notice in full and 2) give the full citation on the first page. It is permissible to abstract these works so long as credit is given. To copy in all other cases or to republish or to post on a server or to redistribute to lists requires specific permission and payment of a fee. Contact Publisher@InformingScience.org to request redistribution permission.

\section{Introduction}

The institute for Community Entrepreneurship and Development (ICED) has a long history of involvement in the practice of community development and social entrepreneurship within the Cree, Black and other minority communities. The visible minority communities account for a significant proportion of the 
populations in Metropolitan areas across Canada (Statistics Canada, 2006). But their community organizations and businesses are small and exist outside the mainstream of Canadian growth and prosperity. They make up the rank and file of what is being increasingly identified as "below the radar" organizations and businesses that operate in the "cracks in the market". Typically, these types of organizations and businesses lack access to the kind of knowledge and updates in knowledge essential to keeping pace with the growth in capacity and ingenuity necessary for making them competitive with the large scale mainstream organizations and oligopolies operating in the national market. In Canada, they are concentrated in the cultural communities and may in some cases form weak social and economic bridges with agencies in the mainstream economy and society.

ICED researchers believe that the gaps in ingenuity and management skills that retard the development of these micro-family based businesses can be removed by addressing two problems. The first involves modifying traditional economics and managements principles and concepts and ways of teaching them so that they are more sensitive to the scale of operations of "below the radar organizations" and businesses entering the national market via these "cracks in the market". The second relate to the use of communication technology for creating a network system of activity centers that receive, process (evaluate, use and update knowledge or ingenuity) and distribute information on a continuous and real time basis to these businesses and organizations. In order to better understand this in a social and economic context, Bayne and Saade have drawn on the concept of the fitness landscape within the framework of a self-adaptive cultural change model. They argue that the fundamental reason for social change and adaptation is to optimize social and economic well being on a continuous and sustained basis. They propose that cultural change and adaptation is facilitated by exchange of goods and information, especially information in the form of "know how or technology". Social and environment sustainability is a bye-product of the search for this optimum.

\section{The Fitness Landscape Model of Social Sustainability}

The fitness landscape model is based on the notion that human existence and the perpetuation of life and the quality of life are dependent on human activity, ingenuity, environmental and other influencing events. A mapping of the total possible outcomes resulting from all human decisions aimed at attaining the best life possible would define a fitness landscape. Such a mapping would have many configurations ranging from relatively smooth undulating to deep canyons and high mountains. By analogy, these represent economic boom and bust, famine, pandemics, floods, storms, earthquakes, wars, degradation of life supporting eco-systems, and the general possible and often unpredictable disastrous consequences of human activity on the biosphere. Thus, the fitness landscape is an environment that offers a range of possible relationships between different groups, organisms, and societies within are special boundaries and constraints. The physical aspects of the landscape itself may change depending on the nature of these relationships (the structure of the interdependencies). The concept of the fitness landscape describes human strategies of reproduction, survival, and the perpetuation and attainment of the highest possible quality of life in an environment. The model provides a framework for studying the struggle of the human species to overcome physical and social barriers to survival, and the role that collaboration and exchange plays in the improvement in life styles.

The two diagrams (figures 1 and 2) adopted from Kobi et al. (2003) illustrate the population space and the belief spaces; the flow of knowledge to the belief space from the people space; and the legitimization and feedback of that accumulated knowledge to influence decision making at the local areas of the population space. Learning at the local level is assumed to be experiential: success-failure oriented or self-adaptive. 


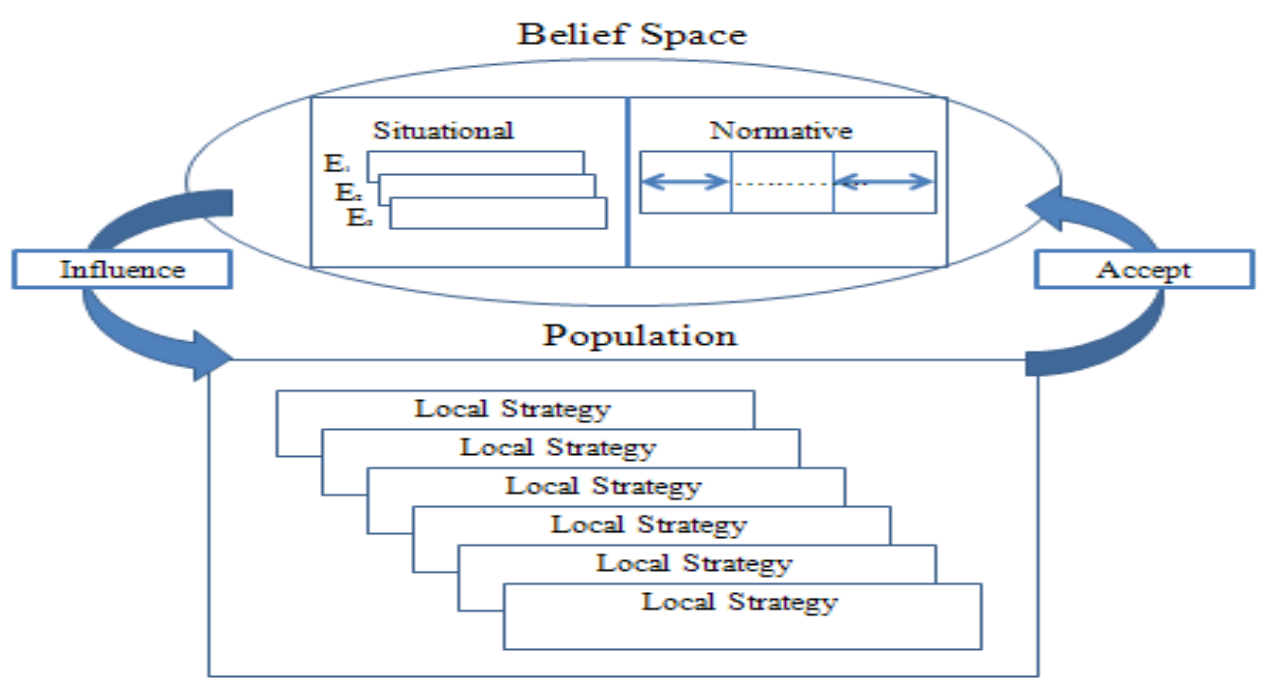

Figure 1. Population space.

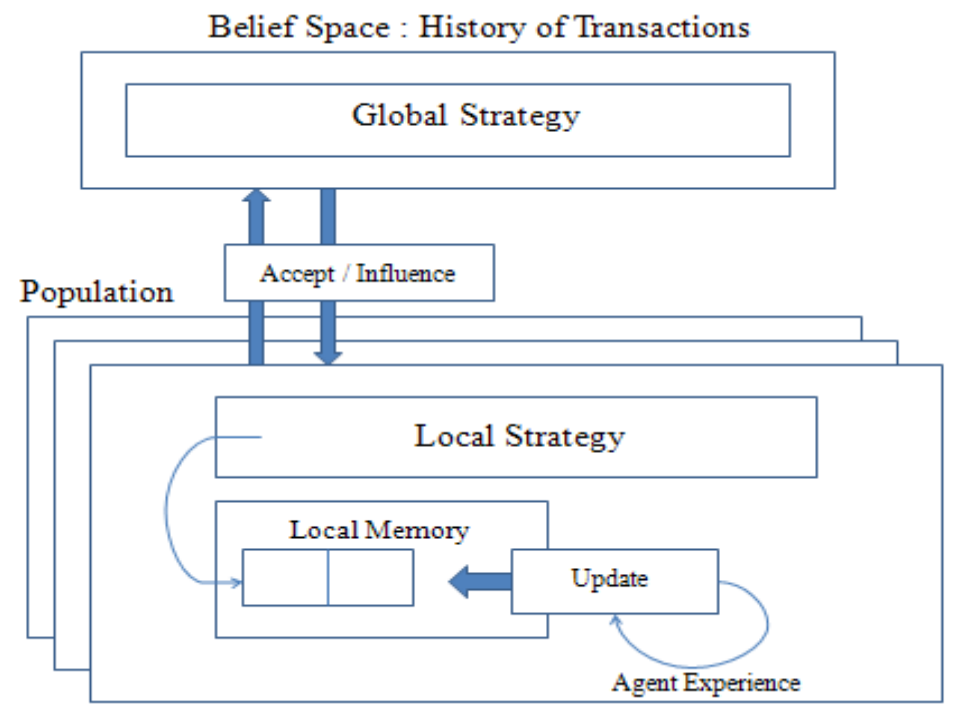

Figure 2. Belief space.

Figure 3 is an adaption of the concepts modeled in figures 1 and 2 to the Canadian fitness landscape. Canada is a subsector of the global biosphere. It consists of subspaces (viewed as ecosystems) supporting and ensuring the perpetuation of life. The Canadian society and economy (fitness landscape) is portrayed as consisting of a private sector producing goods and services, a public sector and a social economy consisting of organizations and agencies as institutional legitimizes and social entrepreneurs. 


\section{Diagram 3}

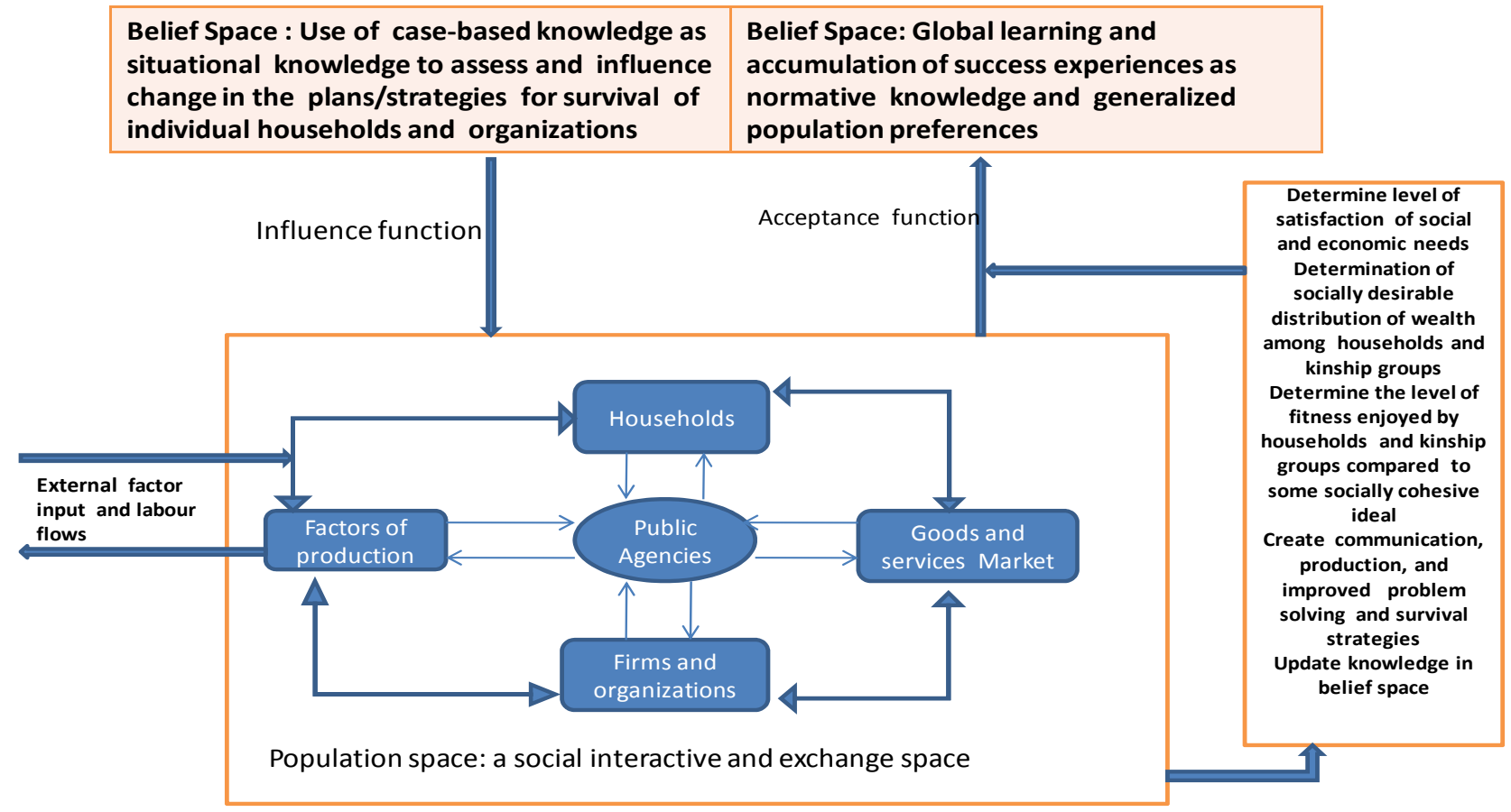

Figure 3. Canadian fitness landscape.

It is an open system that exchanges goods and services, ideas and values, and peoples (social capital) with the rest of the world (other social and economic systems external to it). The system activities are driven by the will of diverse cultural or kinship groups within it to live and be happy. The movement of people into the system (immigrants) is dictated in part by the internal needs of the system (Canada) to provide for the consumption needs (social and material) of its citizens; and maintain inventories (goods, service delivery capacity, ingenuity) that minimize the likelihood of starvation, chaos, and other threats (drastic social and economic hardship) over time. In general, there is a value and belief system in place that supports the view that a fair and equitable distribution of opportunity, goods and services is socially desirable. Immigration is controlled by economic necessities (market size, security, manpower and skills needs) and by the social reciprocity of the society (the Citizens). This reciprocity is reflected in the immigration point system (arguably so), the refugee policies that govern certain types of immigration to the country, and the quality of immigrant settlement policies embedded in a social economy (National institutions and loose local networks of support organizations).

\section{Testing the Proposed Model}

A recent pilot survey was recently conducted by in the Black and visible communities of Montreal. Our survey focused on studying the internal structure of local community networks and determining their strategic purpose in terms of social and economic integration, sense of belonging and being; sense of commitment and responsibility; discovering what are the key factors responsible for success and failure of community structures (centers) in the Black and African minority communities in Montreal; and how we can use this knowledge to develop organizational models that work better and that are sustainable. A central purpose of our research is to facilitate the integration of the most disadvantaged communities into the social and economic decision making processes of the society. 
A questionnaire was developed and a survey conducted. The survey instrument in part followed the guidelines of the GRI Indexfor NGOs and a sense of community index (SCI). The Guidelines identify information that is relevant and material to most organizations and of interest to most stakeholders for reporting the three types of Standard Disclosures:

- Strategy and Profile: Disclosures that set the overall context for understanding organizational performance such as its origin purpose, mission and mandate, strategy, profile, and governance.

- Management Approach: Disclosures that cover how an organization addresses a given set of issues in order to provide context for understanding performance in a specific area.

- Performance Indicators: Indicators that elicit comparable information on the economic, environmental, and social performance of the organization.

The survey also involved in depth tape and video interviews of the principal decision makers in these organizations; as well as reviews of studies and reports of the operations of the organizations in their cultural communities.

\section{Results of Testing the Proposed Model}

Our analysis reveals serious concerns among the leadership and members of these communities about the short life cycles of new organizations and the decay of old structures that provided the early strategies for survival and settlement. The minority status of these communities and their organizations put them on the periphery of the social economy of Quebec and private and political mainstream society. Differences in cultures, exclusion, scale of organizations and enterprises, a lack of knowledge of the customs and traditions of commerce and the labor market, a lack of knowledge of the typography and access to information are characteristics of these organizations. In the language of sense of community theory, a multiplicity of factors which we will classify as social, psychological, demographic, and economic seem to combine in a complex set of ways to create detachment and alienation in many of these communities. One notices differing degrees of inhibited growth and development that seem to be associated with failure of organizational structures and the institutional arrangements within these communities and between them and the larger society. To strengthen these communities it is important to strengthen the community base organizations, help them to develop strategies that make them more dynamic and capable of surviving crises that are the result of exogenous or endogenous forces at work. The question is what are these factors specific to particular communities? How do they interact to cause failure or success? What can we learn from an understanding of the complexity and interdependencies of the factors themselves?

\section{The "Fitness Landscape" Portal}

We have been working on the development of portal whose primary goal is to link community based centers within a communication network. Community centers are capable of filling in their information and knowledge gaps in a more systematic manner. There may be many different centers, each one or clusters of centers addressing a range of problems and issues impacting on the well-being of a community. Figure 4 shows the structure of the portal between the research unit and the proposed centers within the logical framework of a portal. 


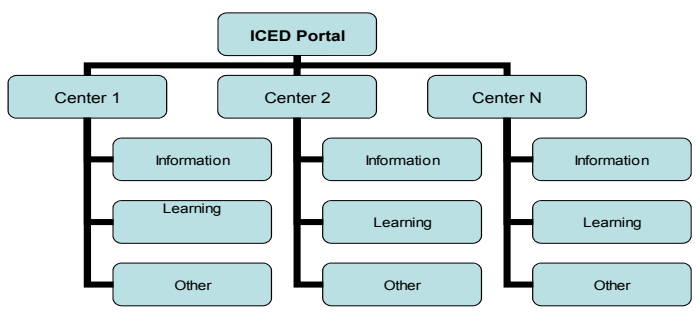

DIAGRAM 4: ICED PORTAL RELATIONSHIP WITH CENTERS

Figure 4. Structure of centers in the portal.

The design of the portal entails the creation of a website structure specific to each center represented in our portal and the mapping of the 'fitness landscape model' as the strategy for these centers to move into higher fitness terrains facilitated by the portal tools. Different elements are included in the portal such as elearning, resource center and other tools. For example, the learning aspect will involve discussions in the portal about social issues; monitoring the progress and displaying outcomes and best solutions; on-line and face-to-face workshops. There will be a realtime feedback loop mechanism created and built into to the site for each Center. All programs and strategies will be subject to a results based management process to determine their efficacy as strategic search rules (procedures). The data collected across all centers can be used to develop a general well-being index for the kinship group. Indexes for each Center will be developed, and updated. This specific and Center-based information can be used to update knowledge in the larger community belief space about the kinship group.

The usefulness of the network dependents on how the outputs (results) of the network are perceived at the individual level; on how effective the network has become in increasing the capacity of their member institutions to achieve and sustain the desired output levels (attain a higher fitness level for their members); and the general general contribution of these achievements to movement to a higher fitness level for the group as a whole.

Using the portal ICED acts from within the belief space to create that balance between the mainstream and the non-mainstream sectors., and to create environmentally more friendly and empathic society. The results are of primary importance to all stakeholders: students, faculty, government, minority communities and society.

\section{Outcomes}

Via the portal, we propose three approaches to closing the "ingenuity gap" and the wealth distribution gap between the minority communities of Montreal and mainstream society. In general the approaches focus on the reduction of unemployment by increasing the social and economic capacities in these communities; and by linking major institutions in these communities to the knowledge systems of mainstream society. In incremental and pragmatic terms ICED will:

1. Organize training workshops based on the innovative instructional approaches and materials that ICED scholars have been developing to assist potential minority business persons start businesses.

2. Organize workshops for the exchange of information and the dissemination of knowledge among community leaders, policy makers and administrators. 
3. Use communication technology methods to create virtual communication centers that will provide a continuous flow of information between the participating community organizations and the University (ICED researchers).

4. Create linkages between the knowledge system at the University and a preselected network of community centers.

5. Create and sustain training workshops for the launching of new businesses and supporting existing businesses and organizations in the minority communities.

It will be necessary to digitize and centralize the rich sources of information available in various community organization files, and make currently inaccessible scholarly resources more accessible to community administrators and potential entrepreneurs on a continuous basis.

We will also put in place protocols to determine the extent to which participating centers and "entrepreneurs" have successfully integrated the training knowledge from our workshops and consultations into the management practices of the organizations; and the trickle down effect to the social and economic life of their membership. This will be accomplished by the implementation of an on line survey based on a Sense of Community and Social Index so that it conforms to the methods used by the GRI Sustainability Reporting Guidelines.

\section{Benefits to the Social Organization}

Participating organizations will receive support during the piloting process through:

- Their participation in workshops and conferences

- guidance materials developed in the pilot studies providing current good practice, new research information and techniques, and sources for further guidance

- information exchanges with peers as well as a community forum to exchange on best practices

- expert input from the ICED Working Group members developing the network centers

- feedback on organization reports based on online reports from the communities and other publics

- visibility as a leader in the reporting field and as a supporter of the ICED social integration index

- being listed on the dedicated public pilot page of ICED website www. Icedportal.com and on communications material related to the network of centers.

- being included and referenced in publications on the Pilot Programme outcomes

\section{References}

1. Population by Selected Ethnic origins, by Province and territory (Statistic Canada, 2006 Census) http://www40.statcan.gc.ca/101/ind01/13 3867_3433-eng.htm?hili_demo26, Visible Minority Population, by Census metropolitan areas (Census 2006): http://www40.statcan.gc.ca/101/cst01/demo53a-eng.htm

2. Third Sector Research Centre (TSRC): Informing Civil Society. Below The radar (BtR). http://www.tsrc.ac.uk/Research/tabid/391/Default.aspx . The centre is a collaborative venture by the University of Birmingham and Southampton, with collaborations fro Middlesex and Kent. This research explores the role, function, impact and experiences of small community action groups or organizations. It include those working at a local level or in communities of interest-such as women's groups or refugee and migrant groups

3. C. Bayne points to many of these small businesses operating in what he calls cracks in the private enterprise sector or market system and attribute their success to the existence of pools of demand that are too small to attract the olygopolists that characterize mainstream markets and the attention of economists and business schools but provide a good but simple and satisfactory life for families. These phenomena seem to be supported by 
the literature on "Degrowth" which is currently emerging as part of the movement for a more socially and economically sustainable world.

4. Ziad Kobi, Robert G. Reynolds, and Tim Kholer, A Multi-Agent Simulation Using Cultural Algorithms: The Effect of Culture on the Resilience of Social Systems, IEEE Congress on Evolutionary Computation December 5-12, 2003, Canberra, Australia.

5. GR1 G3 Content Index: The Global Reporting Initiative (GRI) collects data which measures indicators related to the performance of organizations (profit and non-profit). Global criteria are provided based on efficiency, effectiveness, and sustainability. The organization's own sustainability and business strategy/ provides the context in which to discuss performance. The reporting principles are intended to help achieve transparency in the provision and sharing of information.

6. http://www.kinross.com/media/23991/kinross-grit-tables.pdf; April 12012.

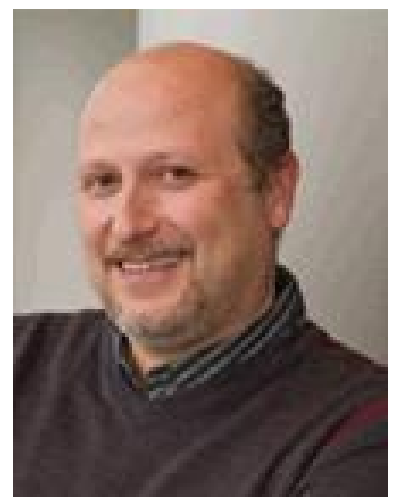

\section{Biographies}

Dr. Raafat George Saade has been teaching in the faculty since 1998. He obtained his Ph.D. in 1995 (Concordia University) after which he received the Canadian National Research Council postdoctoral fellowship, which he completed at McGill University in Montreal. Dr. Saade has published in journals such as Information \& Management, Decision Sciences, and Expert Systems with Applications. His research interests include the development and assessment of information systems, and the supply chain of digital information products.

Clarence S. Bayne 